\title{
Poesía Buenos Aires (1950-1960). Una Revista Argentina de Vanguardia
}

$\mathrm{E}$

STAs líneas tratan de ser, ante todo, la descripción de una revista lite-

raria de vanguardia, Poesía Buenos Aires; ${ }^{1}$ en segundo y subordinado lugar, una evaluación de ciertos aspectos de la misma. El último período de la poesía argentina reconoce pocas instancias unificadoras; una posible vía de aproximación puede ser el examen de algunas de sus revistas más características. Es lo que queremos hacer aquí, dando mayor peso a la aportación de datos, información y citas, que a la formulación de opiniones personales. Aunque estas últimas sean inevitables en cierta medida, han de mantenerse dentro de los límites fijados por una honesta y razonada tentativa de objetividad.

\section{Descripción}

La colección completa" de la revista Poesía Buenos Aires cubre treinta números; el número I del año I corresponde a la primavera de I950; el que lleva la indicación de año once, número 30, es de la primavera de $1960 .^{3}$ En la década transcurrida se observan algunas variantes

1 O poesia buènos aires, como siempre se leyó en la portada de la revista, aunque los textos de sus propios dirigentes oscilen entre una forma y la otra. Cf. númeto 6, verano 1952, p. [1]: "Apuntes para una situación de Poesía Buenos Aires"; número 13-14, primavera 1953-verano 1954, p. [20]: "poesía buenos aires tendrá a bien no devenir institución", etc.

2 Carta de Raúl Gustavo Aguirre a David Lagmanovich, 2 de abril de 1963: "El último número aparecido es el 30 , y no sé si la revista va a continuar". La falta de aparición durante más de dos años consecutivos (situación nunca producida anteriormente) hace suponer que la posibilidad positiva es muy remota.

3 Desde el punto de vista material consta, en realidad, de 26 ediciones, pues son dobles los números 11-12 (otoño-invierno 1953), 13-14 (primavera 1953-verano 1954), 16.17 (invierno-primavera 1954) y 19-20 (otoño-invierno 
en la dirección. Así, desde el número I (primavera de 1950) hasta el número 3 (otoño de 195I) figuran como directores Jorge Enrique Móbili y Raúl Gustavo Aguirre; del 4 al 6 (invierno de I95 I a verano de 1952), Raúl Gustavo Aguirre y Wolf Roitman; del 7 al I5 (otoño de 1952 a otoño de 1954), Raúl Gustavo Aguirre y Nicolás Espiro; del I6-I7 al I9-20 (invierno-primavera 1954 a otoño-invierno 1955), Raúl Gustavo Aguirre; desde el verano de 1955 hasta la primavera de 1956 (números 21 a 24) la dirección nuevamente es conjunta, a cargo de Raúl Gustavo Aguirre y Edgar Bayley; y vuelve a quedar exclusivamente a cargo de Raúl Gustavo Aguirre en su último período, desde el otoño de I 957 a la primavera de 1960 , o sea en los números 25 a 30. Estas variantes, que no hacen sino mostrar con mayor claridad la función que diríase coordinadora de Raúl Gustavo Aguirre, tienen por otra parte relativamente poco que ver con la orientación y el contenido de la revista, caracterizados por una notable homogeneidad.

Además de su aspecto propiamente periodístico, la actividad de los integrantes del grupo Poesia Buenos Aires se hace evidente en una serie de otras formas, entre las que corresponde destacar:

a) Dentro de la revista, pero marcando acontecimientos especialmente señalables, los números II $/ \mathrm{I}_{2}, \mathrm{I}_{3} / \mathrm{I}_{4}$ y $\mathrm{I} 6 / \mathrm{I} 7$, respectivamente dedicados a René Char, a la nueva poesía argentina y a René Ménard, cada uno de ellos consistente en antología, estudio crítico y notas bibliográficas.

b) Las compilaciones Antología de una poesia nueva (I95I), selección, prólogo y notas de Raúl Gustavo Aguirre; Guatemala, 1954, y Diez poemas de amor, 1956, homogéneas presentaciones del grupo.

c) Las ediciones Poesía Buenos Aires, tres de cuyos títulos ya han sido mencionados, y en cuya lista figuran los nombres de escritores y poetas argentinos tales como Raúl Gustavo Aguirre, Jorge Enrique Móbili, Edgar Bayley, Alberto Vanasco, Francisco Urondo, Rubén Vela, Rodolfo Alonso, Elizabeth Azcona Cranwell y muchos otros."

1955). Los números publicados hasta el 19-20, inclusive, o sea de 1950 a 1955, constituyen el volumen $I$, período durante el cual sus sostenedores procuraron mantener una periodicidad trimestral; el volumen II (1956-1960) abarca desde el número 21 hasta el 30 , durante el cual la periodicidad es irregular. Los números del primer volumen, que miden 36 centímetros de alto por 27 de ancho, no tienen paginación; el volumen II, de 19.5 por 14 centímetros, tiene 316 páginas numeradas.

4 He aquí una lista parcial: Raul Gustavo Aguirre, Cuerpo del borizonte, 1951, La danza nupcial; 1954, Cuaderno de notas [prosa], 1957; Edgar Bayley, Realidad interna y función de la poeśá [ensayo], 1952; Jorge Enrique Móbili, Convocaciones, 1951; Alberto Vanasco, Ella en general, 1954; Néstor Bondoni, 
d) La serie "Poetas del siglo veinte", antología en fascículos de poesía contemporánea entre los que aparecieron, por lo menos, los números dedicados a Guillaume Apollinaire, Max Jacob, Paul Ëluard y Carlos Drummond de Andrade.

Volviendo a la revista propiamente dicha, el examen de los treinta números publicados entre 1950 y 1960 indica la existencia de materiales que se agrupan naturalmente en tres tipos:

I. Poemas de poetas argentinos, muchos de los cuales publican por primera vez en $P B A$;

2. Poemas de poetas extranjeros, en traducción si la lengua original no es el español, generalmente con comentarios biográficos y explicativos;

3. Artículos teóricos, históricos o doctrinarios sobre la poesía, de los cuales por cierto interesan especialmente los firmados por los orientadores de la publicación o aquellos que se presentan como nota editorial sin firma.

Revisaremos rápidamente el contenido de cada una de esas imaginarias secciones.

\section{Contenido}

Toda revista literaria es primordialmente un vehículo para que la producción de un determinado número de escritores se ponga al alcance del público; a la vez, y en el caso particular de una revista de poesía, la inclusión de traducciones de poetas de otras lenguas permite igualmente apreciar la actitud básica y la tendencia estética de sus editores. En este sentido, ¿cuál es el contenido de Poesía Buenos Aires?

En lo que se refiere a escritores argentinos, es evidente que los que están presentes con un número mayor y más constante de contribuciones

Trevesia [relatos], 1956; Francisco Urondo, Historia antigia, 1956, Breves, 1958, Dos poemas, 1958; Ramiro de Casasbellas, El doble fondo, 1954; Rubén Vela, Verano, 1954; Rodolfo Alonso, Buenos vientos, 1956; Alejandra Pizarnik, La ullima inocencia, 1956; Elizabeth Azcona Cranwell, La vida disgregada, 1956; Osmar Bondoni, Poemas, 1957; Osvaldo Elliff, Poemas solos, 1957; Leonidas Lambcrghini, Al público, 1957; Manuel D'Ornellas, Conquista del mar y la arena, 1957; Santiago Bullrich, Oda telegráfica a Tenocbtitlán, 1957; Mario Porro, La vigilia y la roca, 1957; Daniel Giribaldi, Agua reunida, 1958. Estrechamente vinculado con la revista parece haber estado el sello "Ediciones Altamar". 
son Raúl Gustavo Aguirre, ${ }^{5}$ Rodolfo Alonso, ${ }^{6}$ Edgar Bayley, ${ }^{7}$ Francisco José Madariaga, ${ }^{8}$ Mario Trejo ${ }^{9}$ y Francisco Urondo. ${ }^{10}$ Figuran también, aunque con menor grado de representación, Juan Carlos Aráoz de La-

5 En Diez poemas de amor (Buenos Aires, Ediciones Poesía Buenos. Aires, 1956), p. 5, figura esta composición de Raúl Gustavo Aguirre (1927), titulada "Como inmenso es el mundo...":

Yo conocía tu rumor en mi alma, y en mi alma eras libre de hacer cuanto quisieras. Yo conocía el sol de tu presencia, y te llevaba en mi alma como el mar, como el viento hubieran querido llevarte. Yo cambiaba tu cuerpo por el mío, yo era la.eternidad.

Al azar te encontraba, una y otra vez, y el mundo era demasiado grande como para retenerte o como para que nuestros destinos se contradijeran. (Y tú, tan parecida al aire de pronto, eras tan libre como yo, y nos cambiábamos sin saberlo, sin nombrarnos, sin descubrirnos la razón de nuestra maravillosa indolencia). Pero esta sombra no durará, no durará.

6 De Rodolfo Alonso (1934) es la siguiente "Aria del perdido", publicada en $P B A(30): 286$, primavera 1960:

dónde encontrar la voz errante

la voz temible y ágil que ilumina la sangre

su sonido es agua en el camino

el resto tiempo y gracia

dónde encontrar esa orilla extraviada

esa región de amor

ese fuego que vive por nosotros

la voz

alguien la oyó para morit

a solas

algo la excede entonces o le falta

algo que aplaza y que restringe

que no nos favorece

hay un abismo al borde del silencio

en lo alto de la voz

vivitemos a merced de su aliento sagrado

7 Edgar Bayley (1919) firma este poema, "El camino", en $P B A$ (27): 179-180, primavera 1958:

Tras ia puerta

se extiende el camino

ese viento

que en la noche abre tu silencio

He tenido tu sueño

húmedo como la hierba del sendero

he tenido tu sombra

$y$ tu paso 
madrid, Omar Rubén Aracama, Elizabeth Azcona Cranwell, Juan Jacobo Bajarlía, Osmar Luis Bondoni, Ramiro de Casasbellas, Nicolás Espiro, Clara Fernández Moreno, Daniel Giribaldi, Hugo Gola, Alberto Lores,

\footnotetext{
He venido caminando

$y$ he reconocido tu puerta

$y$ el árbol junto al río
}

Hay que partir

y llegar mucho más lejos

olvidarse del cielo

y del silencio
Alguien marcha
a lo largo del camino
y tu palabra escapa
al despuntar el sol
como el viejo solitario
que bebe en la mañana su grapa
con las manos vacías

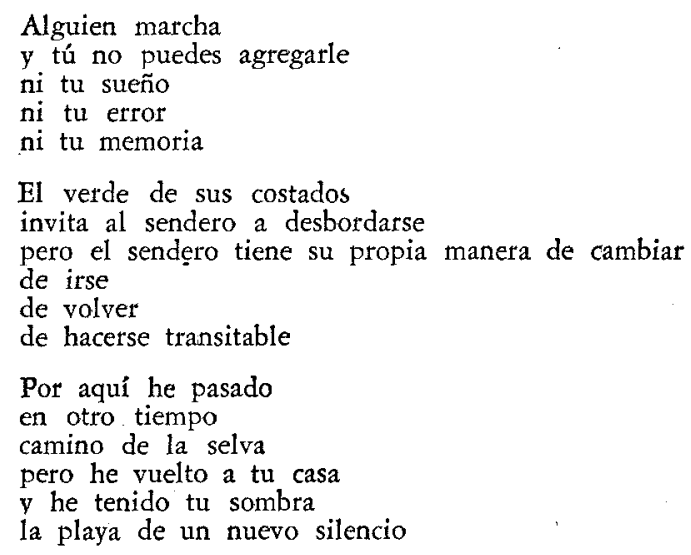

8 Este poema de Francisco José Madariaga (1927), "Arte poética", puede encontrarse en $P B A$ (27):190, primavera 1958:

¿Adoras la criatura que huye vertical y envilecida?

Las doncellas enanas son la bondad de esta fiesta.

Tus hermanitas, las leves miserables estrellas, son desleales al infinito.

Tú no te recuestes más acá de los lenguajes humanos.

Aulla entre los comercios donde la nieve es roja, en las noches de julio cuando hurgamos, sin que nos ate ninguna intención.

9 De PBA (5): [3], primavera 1951, tomamos el siguiente poema de Mario Trejo (1926), titulado "Continuidad":

la ternura progresiva de tus pasos

los pasos opuestos de tu voluntad 
Jorge Enrique Móbili, Enrique Molina, Franco Mogni, Aldo Pellegrini, Wolf Roitman, Alberto Vanasco y Rubén Vela. Igualmente resulta significativa, en virtud de la distancia cronológica respecto del grupo, la inclusión de trabajos de Macedonio Fernández, Ricardo Güiraldes, Oliverio Girondo y Juan L. Ortiz: indicación de una tendencia a la continuidad con ciertos aspectos de la literatura nacional que no siempre caracteriza a los movimientos literarios de vanguardia.

La lista de poetas extranjeros (cuyos trabajos se presentan en traducción española, acompañada de una nota biográfica casi siempre) es abultada, y nos permite apreciar la existencia de distintos grupos:

los de lengua francesa: Guillaume Apollinaire, Paul Eluard, René Char, T'ristan Tzara, André Bréton, René Ménard, Pierre Reverdy, Francis Ponge, Francis Picabia, Alain Bosquet, Jacques Prévert;

los de lengua inglesa: Dylan Thomas, E. E. Cummings, Hart Crane, Wallace Stevens, Emily Dickinson;

los de lengua portuguesa: Carlos Drummond de Andrade, Milton de Lima Sousa, Murilo Mendes, Heitor Martins, Ary Xavier, Egito Gonçalves, António Ramos Rosa, Fernando Pessoa;

los hispanoamericanos: César Vallejo, Pablo Neruda, Vicente Huidobro, Braulio Arenas, Alfonso Cortés, Otto Benítez.

Hay otros poetas representados, por supuesto: desde Catulo hasta

la voluntad crispada de tu cuerpo
el cuerpo emancipado de tu sombra
la sombra socavada de tu boca.
la boca cautiva de tu sonrisa
la sontisa descortida de tus muslos
los muslos asomados de tus mañanas
la mañana aventurada de tu voz
tu voz inventora de mi nombre
el nombre esparcido de tu existencia
la existencia auspiciosa de tus manos
las manos estacionarias de tu ternura

1.0 En PBA (22):59, otoño 1956, figura esta composición de Francisco Urondo (1930). Se llama "Bar 'La Calesita" ":

Este es el fondo de un bar. Es un lugar parecido a una cueva, donde uno se sienta, bebe y ve pasar hombres enrarecidos por distintos problemas. Es una gran linterna mágica.

Es una gruta retirada del mundo que cobija sus criaturas. Uno se siente allí ferozmente feliz.

Acaba de aparecer el primer hombre, apenas ha aprendido a caminar, aún no sabe defenderse.

El hombre sonríe y llora y sigue la fiesta. 
Pasternak. Pero los mencionados bastan para dar una idea de la variedad de materiales publicados por esta revista en sus once años de vida, así como de las tendencias predominantes en los grupos de avanzada de la vida literaria argentina durante ese mismo período. No resulta arriesgado inferir, en ese sentido, que es éste en la Argentina uno de los grupos poéticos de vanguardia de más perspicaz interés por la literatura contemporánea de otras lenguas. La lista de lecturas extranjeras - y las correlativas posibilidades de renovación formal y temática- frecuentadas por los integrantes de Poesía Buenos Aires coinciden, seguramente, en alguna medida con las preferencias o intereses de otros grupos. Pero dentro de mi país resaltan estos hechos: a) ningún otro grupo, en cuanto tal, realizó un estudio tan detenido de René Char, René Ménard o Pierre Reverdy, por ejemplo; b) ningún otro grupo presentó parecida atención, similarmente, a la poesía de Cummings o a la de Dylan Thomas; y c) la promoción del conocimiento de la poesía actual en lengua portuguesa (en Brasil y Portugal) es una sostenida actividad que estos poetas casi han monopolizado, pues los demás sectores de la vida literaria argentina mantienen una incomprensible incomunicación con las letras actuales de ese ámbito lingüîstico.

\section{Doctrina}

Hemos dicho que tanto en la orientación como en el contenido de esta revista hay notable homogeneidad. ¿Qué orientación, qué doctrina? Hay varias respuestas; $y$, como suele ocurrir en las publicaciones dominadas por un espíritu juvenil, los propios integrantes del grupo se contradicen a veces. En el número inicial, Jorge Enrique Móbili anunciaba: "Nos proponemos dar a la poesía un aire de novedad, de lumbres arriesgadas, de continuidad, de madurez, de absoluto frente a todas las actividades subyacentes del pensamiento humano"; ;1 cualquiera sea el significado de ese párrafo, no parece ser el mismo el de esta concisa declaración de Raúl Gustavo Aguirre: "La poesía no tendrá explicación para nadie". ${ }^{12}$

El punto de vista de los demás, de los que miran desde fuera, difícilmente puede ser más claro. Al ubicar a Bayley entre aquellos que él llama "estos superrealistas", por ejemplo, Juan Carlos Ghiano"13 viene

11. $P B A$ (1):[2] primavera 1950.

12 PBA (1):[2] primavera 1950.

13 Juan Carlos Ghiano, Poesía angentina del siglo XX, México-Buenos Ai- 
a reducir toda la acción de Poesía Buenos Aires a una suerte de vano intento en el que la doctrina sobrepasa en peso específico a la teoría: "han teorizado con abundancia sus intentos, sin que estas poéticas cubran los desacuerdos de la propia obra, o los balbuceos expresivos que todavía no conciertan estilos, amparándose en la justificada inconexión de las intuiciones y en el avasallamiento de las posibilidades rítmicas y estróficas", dice. Menos aún significan estos poetas para Roberto Hurtado de Mendoza:14 "El de los jóvenes de este grupo es más una tarea de ejercicios poéticos antes que trabajo fundamental de poesía. Traída de los cabellos, han pretendido asimilar una posición estética a un medio donde lo primero que se debe hacer es escribir y luego inventar la estética correspondiente [...]. Hasta el presente [...] la calidad creadora, innovadora y el mensaje de sus poemas, no revisten el mínimo propicio como para adjudicarles un sitio real, valedero, dentro de la poesía joven nacional". "Menor tradición nacional" señala igualmente Enrique Anderson Imbert, yay quien también caracteriza brevemente: "Antes se había hablado de creacionismo: ahora se hablará de invencionismo". Carlos Manuel Muñiz se pregunta con incrédulo asombro, en el título de un artículo: ${ }^{16}$ "Otra nueva generación poética?", pero la duda pasa a ser desconcierto total en el caso de Roberto F. Giusti, quien, escribiendo en I960, todavía no está seguro de la existencia de una "generación del 40" $y$, por supuesto, parece creer que de allí en adelante el panorama es aun más confuso:17 "Insisto en que no es posible $[\ldots]$ discriminar en todos los casos con acierto los valores más recientes, admito que algunos ya en sazón; unos, marcadamente afiliados a ciertas tendencias, o neorrománticos o superrealistas, otros más difíciles de clasificar. Se pretende hablar de una generación 'de 1940', pero otras 'generaciones' -apenas, si promociones o reducidos círculos- atropellan para ocupar el escenario y desalojar a quienes acaban de llegar". Escasa claridad hay también

res, Fondo de Cultura Económica, 1957 (Colección Tierra Firme, 65). La cita es de p. 257.

14 Roberto Hurtado de Mendoza, "Análisis de la poesía argentina joven (II)", en Semirrecta, 1(4):17-20, feb.-mar. 1953; cita, p. 18.

15 Historia de la literatura bispanoamericana; II., Época contemposánea, México-Buenos Aires, Fondo de Cultura Económica, 1961 (Breviarios, 156). Cita, p. 300 .

16 Cf. Cíudad, (1):88, primer trimestre de 1955. Se trata de un comentario al acto poético "Presentación de la nueva poesía argentina. La generación última", realizado por $P B A$ en septiembre de 1954, y presentado por César Fer * nández Moreno.

117 "Ciento cincuenta años de poesía", en Comentario, 7(25):50-67, 2? entrega 1960. La cita es de pp. 65-66. 
en las apreciaciones de David Martínez:18 "Como reacción a los gustos tradicionales de los poetas estudiados anteriormente, en un grupo cada día más numeroso, se está operando, por un lado, un decidido avance hacia las escuelas poéticas europeas que estuvieron en auge hace más de veinte años - surrealismo, creacionismo-; y por otra parte, un decidido acercamiento a las más eminentes voces de nuestros días, también europeas y algunas americanas: Eliot, Pound, Luc Deaunes, John-Perse [sic], René Char, Vallejo; y, en menor grado, pero evidente: Ungaretti, Quasimodo, y el español Vicente Aleixandre. ${ }^{19}$ Estos nombres, sumados a los de Bréton, Ëluard, Huidobro, y en su muy pocos, Aime Cesaire, marcan la órbita en que se mueve el azaroso tránsito de los poetas aquí reunidos";20 seguidamente afirma que Poesía Buenos Aires "nuclea a los hombres más salientes de esta promoción: Edgar Bayley, Raúl Gustavo Aguirre, Mario Trejo, Alberto Vanasco, Francisco Urondo, entre los de mayor proyección", y encuentra en ellos cierta "dilección por resucitar o adueñarse de estéticas que hace años han dejado su saldo positivo o negativo". La alternativa surrealismo-creacionismo (invencionismo) es también señalada por Horacio Jorge Becco:21 "Dos corrientes monitoras han estado gestándose con una vivencia que perdura en nuestros días, el invencionismo y el surrealismo. Fuera de ellas con toques más riesgosos, delimitan los confiados en su propia voz, aquellos que conocen la intimidad de los secretos, que son transparentes al latido como verdad, que esperan esforzándose las palabras hechas aventuras" [?].

Como suele ocurrir, hace falta tomar cierta distancia para ver con mayor claridad. Preocupado por establecer el lugar que corresponde a cada grupo en la cronología de la evolución literaria argentina, el crítico uruguayo Emir Rodríguez Monegal"22 subraya, ante todo, que "la llamada generación de 1940 (ó 4I) es, en cierto sentido, nada más que un

18 "Informe sobre la nueva poesía argentina (1930-1958)", Universidad (Santa Fe. Argentina), (38):179-203, jul.-dic. 1958. El mismo material figura como introducción a las secciones de: David Martínez [comp.], Poesía argentina actual (1930-1960), Buenos Aires, Ediciones Culturales Argentinas, 1961. Los trozos citados se encontratán en pp. 174-175.

I9 Compárese esta lista de influencias -en orden y contenido- con la que proporciona Anderson Imbert, ob. cit., p. 299-300: "Eliot, Pound, Luc Deaunes, St-John Perse, René Char, Vallejo, Ungaretti, Aleixandre".

20 Que son Rodolfo Alonso, Juan José Ceselli, Héctor Eduardo Ciocchini, Juan Carlos Ghiano, Joaquín O. Giannuzzi, Alberto Girri, Fernando Guibert, Eduardo A. Jonquières, Francisco José Madariaga, H. A. Murena, Osvaldo Rossler, Emilio Sosa López, Mario Trejo, Alberto Vanasco.

2.1 "Evolución y tendencias de la poesía argentina actual (1950-1960)", Ficción, Buenos Aires (24/25):37-42, mar.jun. 1960. Ver p. 38.

22 Emir Ròdríguez Monegal, El juicio de los parricidas; la nueva generación argentina y sus maestros, Buenos Aires, Deucalión, 1956. Cita: p. 88. 
grupo de epígonos de la de I925", y que "es el grupo del 50, en realidad, el que asume toda la responsabilidad de plantar, desde las raíces mismas, el nuevo sistema de vigencias; que ataca buscando llegar al fondo; que remueve las estructuras externas; que se aparta de los caminos trazados; que pone en cuestión la literatura, empezando por el concepto mismo de la literatura y concluyendo con el concepto de escritor y su validez social. Para este grupo hay que empezar de la nada, a partir de cero (como dice el título de una de sus revistas). Y es esta actitud lo que lo define como generación nueva: una doble actitud visible en la crítica y en la creación". Todo ello, dicho genéricamente del grupo de escritores que surge coherentemente a la vida literaria argentina alrededor de I950, puede afirmarse aun con mayor exactitud de Poesía Buenos Aires. Y la otra observación atinada es la de Alfredo A. Roggiano, quien al estudiar las tendencias de la nueva poesía argentina ${ }^{23}$ define la actitud de Raúl Gustavo Aguirre, animador del grupo, como una tendencia a la "integración social y humana de la poesía y del poeta", rescatando la polémica del ámbito exclusivamente libresco en que se debatía en el caso de varios de los críticos citados.

¿Superrealismo? ¿Invencionismo, versión remozada del creacionismo? Veamos. La poética del grupo Poesia Buenos Aires, como bien lo señala Roggiano, ${ }^{24}$ está formulada, inicialmente al menos, en el prólogo de Aguirre a la Antologia que constituye una primera expresión colectiva del movimiento. ${ }^{2 \sigma o}$ Allí, después de trazar un esquema de la poesía de este siglo en torno de "la prescindencia, en el lenguaje poético, de todo elemento anterior a la imagen, de todo sustento previo del poema", a través del superrealismo y el creacionismo, Aguirre rebautiza a este último - siguiendo a Edgat Bayley - con el nombre de invencionismo: su significación, afirma, "no reside tanto en haber descubierto un nuevo tipo de imagen (aunque sí lo ha destacado, diferenciado netamente), como en lo que significa como toma de conciencia del problema desde un punto de vista que representa la culminación del desarrollo del lenguaje poético". Por otra parte, dice, desde fines del siglo xxx asistimos a una evolución en la significación de la poesía, desde "una vaga ocupación de orden estético" hasta "una manera de vivir". "La poesía ya no es una actividad aparte"; ella "intervendrá en la construcción de un mundo

123 "Situación y tendencias de la nueva poesia argentina", tirada aparte de la Inter-American Review of Bibliograpby, 13(1):[1]-29.

24 Ob. cit., p. 26.

125 "Para un concepto actual de la poesía", prólogo a Antología de una poesía nueva, selección, prólogo y notas de Raúl Gustavo Aguirre, Buenos Aires, Ediciones Poesía Buenos Aires, 1952. 
nuevo", ya que "la condición esencial de un poema es la de ser, perpetuamente, de alguna manera actual, de alguna manera útil para la vida de los hombres".

Edgar Bayley había explicado por los mismos años que su invencionismo no implicaba ningún hermetismo, pues "la disposición de espíritu merced a la cual el poema nace, la experiencia dramática que lo nutre, es la misma que se pone en juego en todas las formas de pensamiento o de expresión de los individuos o de las colectividades"; ${ }^{26}$ pero al afirmar también que el lenguaje poético es aquel en donde "la palabra entra en relaciones que, en vez de reducir o encerrar su poder poético, como en el discurso lógico, tienden a liberarlo" $[\ldots]$ y "es en este acto de liberación creadora de la energía emocional de las palabras, donde parece residir el proceso interno de la poesía", ${ }^{27}$ corría precisamente el riesgo de desplazarla no hacia el polo hipervital, sino hacia el hiperintelectual. De la disyuntiva sale advirtiendo que "tal invención, si no aparece inmersa en un tono vital coherente y perceptible por todos, será sólo una acumulación sin sentido de palabras; a lo sumo, un juego sin trascendencia". Esta búsqueda de la comunicación con el prójimo, este sentido comunitatio de la poesía, está presente en una larga serie de documentos, algunos de ellos presentados como notas de la dirección, otros firmados por Raúl Gustavo Aguirre. Citamos o glosamos sólo los que creemos más importantes. "El movimiento que sustentamos - la poesía-sólo puede significar el desplazamiento, hacia valores verdaderos, de los motivos de la convivencia humana y de las formas en que éstos se concretan"; "La identificación de la poesía y la vida es un proceso universal y trascendente". ${ }^{28} \mathrm{El}$ poeta, por su parte, siempre está vinculado con la realidad de alguna manera (esto responde a la acusación de extranjerismo inmediatamente lanzada desde los reductos del nacionalismo literario), "y tanto más cerca de ella cuando trasciende las superficies exteriores y las formas para estarlo de un modo más íntegro, aunque menos aparente, cuando ejercita todos los medios de que dispone el ser humano para adquirir una imagen auténtica del mundo".29 $\mathrm{Y}$ finalmente: "Creemos que la Poesía no sólo es decisiva aventura por el conocimiento, sino - y en tanto- que entraña una relación en la que está im-

26 "Invencionismo", $P B A$ (1):[4], primavera 1950.

27 "Realidad interna y función de la poesía", $P B A(6):[3-4]$, verano 1952, y (7):[7-12], otoño 1952 .

28 "Apuntes para una situación de Poesia Buenos Aires" [nota de la di. rección], $P B A(6):[1]$, verano 1952.

29 Raúl Gustavo Aguirre, "Presencia de la realidad en la poesia", $P B A$ (9):[1-2], primavera 1952 . 
plicada cualquier realidad: un vínculo esencial entre los hombres, o -mejor aún-la experiencia de ese vínculo [...]. Si en este tiempo de bombas atómicas, persuasores ocultos y policía generalizada no tuviésemos la evidencia de que a la palabra del hombre le es posible abrir al hombre el camino de lo que Heidegger llama "su casa" y Keats su "belleza-verdad", podría aparecer esta tarea como una inútil tentativa de huir, negándola, de una realidad que no of rece otra alternativa que la rebelión o el sometimiento". ${ }^{30}$ No se trata entonces, solamente, de adquitir un lenguaje poético contemporáneo, para la vanguardia como es entendida por los protagonistas de Poesía Buenos Aires; se trata también de hablar dẹsde el nivel del hombre y en función del hombre integro, no de una parte o un segmento de él. El compromiso vital de Poesía Buenos Aires, por encima de las características que llamaríamos estilísticas en sentido estricto, pasa así a ser un rasgo dominante en todo intento de caracterización.

\section{Trayectoria}

Es toda una década, y aun algo más, lo que cubre la revista iniciada en la primavera de r950 por Jorge Enrique Móbili y Raúl Gustavo Aguirre. Durante ese período, naturalmente, la publicación no acaparó todo el movimiento poético argentino; pero una parte considerable de ese proceso sucedió en relación de afinidad o antagonismo con la misma. La trayectoria, la actitud y - en definitiva - la situación de Poesía Buenos Aires pueden quizá surgir de la exposición de algunos hechos ordenados cronológicamente.

I. Si partimos de la ya mencionada Antologia de 1952, encontramos en las palabras preliminares la siguiente declaración, definitoria del criterio sustentado para incluir a los poetas representados: "Esta poesía distinta puede ser encontrada tanto en aquellos que han comprendido el difícil mensaje del surreliasmo como en aquellos que siguen el llamado de Vicente Huidobro o los que, en las posibilidades de la invención poética, buscan expresar su convicción en la magnificencia del destino humano" (p. 25). Bajo la palabra "surrealismo", una importante nota de pie de página expresa: "Después del fracaso de una generación que lo tomó como juego, no sabiendo estar a su altura ni comprenderlo,

30 Raúl Gustavo Aguirre, [Nota a un poema de Dylan Thomas], PBA (30) :315-316, primavera 1960 . 
perdiendo así la oportunidad de realizar la renovación poética que ha permitido a Chile y a Brasil estar hoy a la cabeza de la poesía americana". En otras palabras: los vanguardistas de 1952 reaccionan contra los vanguardistas de 1922 , contra los "martinfierristas", y les acusan de no haber llevado a cabo en forma cumplida el programa de renovaciones establecido. Este tipo de recriminación generacional es, creo, bastante frecuente en la historia literaria hispanoamericana.

2. Durante el mismo 1952 la dirección polémica cambia, y ya no se trata de una generación anterior sino de los reparos de los contemporáneos, algunos de ellos evidentemente situados fuera de la poesía. Lo mismo que otros movimientos vanguardistas, Poesía Buenos Aires tiene que defenderse contra quienes sostienen la necesidad de un mayor y más explícito vínculo localista, autóctono, posiblemente indianista o folklórico hispanoamericano. En un artículo ya citado"s1 se lee: "Buenos Aires, ciudad de estructura oceánica, sólo puede producir - quiérase o nouna cultura universal"; "Una manera de ver que limite esta cultura al elemento autóctono o al hispanoamericano sólo puede tener como resultado la sofocación de otras fuerzas vitales que sostienen su configuración espiritual. Producto de este localismo ficticio sería un arte igualmente estéril y amanerado". Y $Y^{Y}$ también, en actitud muy similar a la que adoptaría un escritor en nada vinculado a los miembros de este grupo, Jorge Luis Borges, ${ }^{32}$ se expondría la idea de lo nacional "por añadidura": el arte es nacional por añadidura, se dice, "porque lo es en tanto auténtico, salido de una situación vivida en determinado lugar del tiempo y del espacio, cuyo sentido se ha sabido penetrar". Lo mismo, al año siguiente:33 "¿Por qué preocuparnos entonces si el poeta está o no vinculado con la realidad? Siempre lo está de alguna manera, y tanto más cerca de ella cuando trasciende las superficies exteriores y las formas para estarlo de un modo más íntegro, aunque menos aparente, cuando ejercita 1952.

31. "Apuntes para una situación de Poesia Buenos Aires", (6):[1], vetano

32 Cf. Jorge Luis Borges, "El escritor argentino y la tradición", en Discusión, Buenos Aires, Emecé, 1957; p. 162: "Por eso repito que no debemos temer y que debemos pensar que nuestro patrimonio es el universo; ensayar todos los temas, y no podemos concretarnos a lo argentino para ser argentinos: porque o ser argentinos es una fatalidad y en ese caso lo seremos de cualquier modo, o ser argentino es una mera afectación, una máscara". El artículo de Borges se publicó inicialmente en Sur, 232, en.feb. 1955, pp. 1-8.

33 Raúl Gustavo Aguirre, "Presencia de la realidad en la poesía", PBA (9): [1-2], primavera 1953 . 
todos los medios de que dispone el ser humano para adquirir una imagen auténtica del mundo".

3. Pero así como había en los hombres del 50 la intención de destacarse netamente de los que habían sido renovadores de la poesía hispanoamericana en la década del veinte (con excepciones tales como $\mathrm{Ne}$ ruda, Vallejo y Huidobro), también hạbía la de distanciarse con claridad de un grupo mucho más próximo cronológicamente, el que en la Argentina se llama habitualmente "generación del 40". Uno de los colaboradores de Poesía Buenos Aires, Rubén Vela, llega virtualmente a sostener la inexistencia de tal grupo, al afirmar ${ }^{34}$ que lo que importa es, precisamente, "fijar definitivamente la correspondencia que existe (aún en sus evidentes contradicciones) entre el espíritu del 25 y el del 50 , en cuanto a impulso, porque más allá de esa generación de Martín Fierro, llegamos a ésta, en un salto profundo que excluye a los grupos que denominariamos reacción del 40 , esa desteñida congregación de poetas que crecieran a la sombra de Machado, Lorca y Hernández, esa poesía de los grupos del 40 que pretendió crear por repetición una gran arquitectura poética, bello edificio externo pero desprovisto de ese sustrato vital que caracteriza a la poesía verdadera".

4. A riesgo de persistir demasiado tiempo en la cita de actitudes de negación, quiero mencionar que el deslinde generacional está también explícitamente formulado en las consideraciones incluidas en el número de la revista que examina o presenta los "poetas de hoy" de Buenos Aires en r953. Allí se lee: ${ }^{35}$

Por otra parte, queremos insistit en no reconocer dentro de los dominios de la poesía a los siguientes supuestos: a) El de las forma retóricas clásicas concebidas apriorísticamente, es decir, como ejercitación verbal de la que pueden llegar a obtenerse resultados felices dentro de un juego de convenciones generalmente admitido. Actitud superficial que implica una parcialización del trabajo creador y un artificioso retorno a épocas donde estas formas eran expresión natural y legítima. b) El de la invalidez, la angustia sin salida, la miseria espiritual del hombre, tema que se origina

34 Rubén Vela, "Realidad de la nueva poesía argentina", Correspondencia, (2) :[42-44], oct. 1956 .

35. "Poetas de hoy: Buenos Aires, 1953", PBA. (13/14):[1], primavera 1953-verano 1954. 
sea en un superficial contacto con el existencialismo, sea en la influencia de poetas del tedio y el refinamiento burgués (Baudelaire, Rilke, Eliot, etc.). Esta escritura de desaliento, de suciedad, de postración, de caos, de patología, nada tiene en común con el sólido y viril - y a veces irónico- estoicismo que sería, cuando menos, la traducción poética de un mal semejante, si es que realmente fuera experimentado por el poeta. c) El de la poesía adjetivada (social, popular, mística, etc.), en cuanto ella significa una postura literaria y no, como debiera ser, un compromiso vital asumido hasta sus últimas consecuencias (Maiakovski, García Lorca, Vallejo) o: una intimidad real, de cultura, de lengua y de espíritu, con el pueblo (Píndaro, Juan Ruiz, Villon, Carlos de la Púa).

Naturalmente, sería fácil encontrar los posibles destinatarios de estas: especificaciones. Quien lea estas líneas con la adecuada perspectiva sospechará que el primer inciso, por ejemplo, quiere decir Jorge Vocos Lescano o Carlos Viola Soto; el segundo, por ejemplo Emilio Sosa. López o H. A. Murena; el tercero, por ejemplo Mario Jorge de Lellis, Fernando Guibert o algunos otros.

5. En 1954,36 en fin, Poesia Buenos Aires anuncia que "en el curso de estos años, con la aparición de nuevos poetas y la consolidación de una actitud de espíritu y vida", la revista "ha ido cobrando, aun sin: proponérselo, el carácter de una expresión de movimiento". Sin definir los límites de tal movimiento, se informa alli a los lectores que el ritmo de las entregas siguientes habrá de reflejar esta "situación de hecho, a la que sería difícil sustraerse". La verdad es que, para entonces, la. publicación ya ha crecido en número de colaboradores $\mathrm{y}$, como allí se reconoce, también en homogeneidad; y el caso es que la declaración citada significará lo opuesto de lo que normalmente se creería: en resumen, aun mayor flexibilidad para el futuro. El "movimiento" de que habla Poesía Buenos Aires se contiene dentro de líneas muy amplias; y los números de su último período, muy importantes por la calidad de las colaboraciones incluidas, tienden sin embargo a prestar atención mucho menor a las actitudes polémicas. De esta manera se cumple la trayectoria señalada con todo acierto por César Fernández Moreno:" "Sus columnas y sus ediciones anexas [...], a partir de una posición inicial

36 "Hoja de ruta", PBA (15): [8], otoño 1954.

37 En: Rafael Alberto Artieta (dir.), Historia de la literatura argentina, $\mathrm{t}_{\text {. }}$ IV, Buenos Aires, Peuser, 1959. 
invencionista, se han ido flexibilizando hasta canalizar y recoger lo mejor de la poesía argentina después de la generación del $40^{\circ}$.

Esa es, a mi modo de ver, la más importante aportación de Poesía Buenos Aires a la literatura argentina: el haber procedido con rigor (en cuanto a la calidad) y con amplitud de criterio al mismo tiempo, hasta constituirse en una suerte de foro o tribuna insustituible para apreciar el desarrollo de la poesía de vanguardia en esa década crucial del 50 al 60. La mayor exposición a los poetas de otras literaturas, y muy especialmente a los de otras tierras de América, es también una de las ventajas notables $\mathrm{y}$ duraderas de esta publicación. El no haber incluido un número suficiente de poetas del interior de la Argentina es una de sus evidentes limitaciones, porque la cultura argentina ya nunca más equivaldrá a la cultura de la ciudad de Buenos Aires. De todos modos, al menos por algunas de las razones apuntadas, los estudiosos de la literatura hispanoamericana del siglo $x x \mathrm{x}$ deberían formular a la revista de Raúl Gustavo Aguirre el más simple y a la vez el más elocuente de los reconocimientos: gracias por existir.

David Lagmanovich

Wasbington, $D . C$. 